\title{
Attachment Theory and the Social Work Curriculum
}

\author{
Timothy Page \\ Rhonda Norwood
}

\begin{abstract}
Attachment theory, as developed by Bowlby and Ainsworth, represented a major departure from the current theories of human development of the time, particularly in its rejection of the major tenets of psychoanalytic theory and its integration of core ideas from evolution theory and cybernetics (Ainsworth \& Bowlby, 1991). Attachment theory posits that a foundational human instinct, the desire to achieve safety and protection through proximity to a protective figure, is responsible for the formation of a special class of life-long affectional bonds, referred to as "attachments." Emotional security is derived to a great extent, according to the theory, from experience with caregivers who are consistently responsive to the developing infant's expression of attachment behavior toward them. Forty years of empirical research has shown that attachment is a universal characteristic that predicts children's development of cognitive and social competence, emotional regulation, and positive self-image (Weinfield, Sroufe, Egeland, \& Carlson, 1999). Social work educators are currently challenged to better integrate the findings of attachment research into their curricula to reflect more the current state of developmental science.
\end{abstract}

Keywords: Attachment theory, social work theory

\section{HISTORICAL CONTEXT OF THE DEVELOPMENT OF ATTACHMENT THEORY}

The creation of attachment theory, beginning with the work of John Bowlby (19071990) and, later, Mary Ainsworth (1913-1999), was, like all great ideas, as much a product of new intellectual currents as it was an explicit rejection of the old ideas it was intended to replace. Bowlby, a British practicing psychiatrist at the Tavistock Clinic in London, was the originator and major theorist, while his colleague, Ainsworth, an American developmental psychologist, extended and provided the first empirical support for the theory with her observational studies in Uganda and later in the U.S. Bowlby developed his ideas about infant development within the intellectual context of mid- $20^{\text {th }}$ century London, where current ideas about child development were dominated by Freudian thought. He diverged from Freudian theory in many important ways, none more so than in his emphasis on the importance of actual experience to human development (Bowlby, 1982). The classical psychoanalytic model was based on a concept of a unique form of internal psychic

Timothy Page, Ph.D. is an associate professor and Rhonda Norwood, MSW is a doctoral candidate at Louisiana State University School of Social Work, Baton Rouge, LA 70803.

Copyright $^{\oplus} 2007$ Advances in Social Work Vol. 8 No. 1 (Spring 2007) 30-48. Indiana University School of Social Work. 
energy, distinguished from physical energy, as the motivating force for human development, conceived of primarily as psycho-sexual drive energy (Greenberg \& Mitchell, 1983). This conceptualization of internal psychic development, the socalled "hydraulic model," was derived from ideas current in $19^{\text {th }}$ century physics concerning the process of accumulation and discharge of energy within closed systems, the goal of which is a state of relative quiescence. In Freud's view, through the process of build-up and discharge of psychic energy, and especially the pleasures and frustrations associated with it, internal fantasy is created, characterized by the sexual nature of drive energy. These fantasies were presumed to be (especially as expressed in Freud's earlier works) even more important to the course of human development than the infant's actual experience. The classical Freudian view, then, is one that stresses the importance of internally derived developmental processes (Bowlby, 1982).

Bowlby replaced the Freudian notion of instinct as psycho-sexual drive with a model of instinct that depended on the interaction of organism and environment, among the earliest and most fundamental of which is the instinct to achieve and maintain physical proximity to a known individual perceived to be protective in times of distress, the foundation for attachment. The motivations associated with attachment, therefore, were seen as fundamentally social in nature, oriented toward building and maintaining close relationships from the time of birth. In this respect, Bowlby's ideas reflected the revisionist school of psychoanalytic theory, object relations theory. He differed from these theorists, however, in several important ways, beginning with his assertion that the instincts and motivations most important for human development are those having to do with the need for safety and protection.

Bowlby also took exception to the theories postulated by some of the most prominent object relations theorists, in particular, those of Melanie Klein and Margaret Mahler, who represented very diverse views within the larger object relations school. He rejected Kleinian theory primarily because of her emphasis on the importance of aggressive and destructive impulses, and the primary significance for the course of development of the child's internal mental representations produced by these impulses. He also rejected Mahler's theory of separation-individuation, starting with her postulation of a phase of "symbiosis," where the infant has no sense of differentiation from the caregiver, as well as the notion that object permanence is acquired only in the third or fourth year. These ideas were not then and, especially as seen in the light of contemporary research, are not now reflective of what is known about the developing infant's perceptions and capabilities (see National Research Council, 2000).

Also, unlike Freudian theory, Bowlby created attachment theory on a foundation of systematic observations of children in their interactions with caregivers. One of the strongest influences on the thinking of Bowlby and Ainsworth was their colleague at the Tavistock Clinic, social worker James Robertson. In several influential films, Robertson observed children in hospitals and orphanages, recording their protest and grief reactions in conditions of confinement, separated from their parents (Robertson \& Robertson, 1959). This early work helped to establish some of the central tenets of attachment theory, that children's intense reactions to pro- 
longed separation from or loss of their primary caregivers indicate that a strong emotional bond exists between them, which begins in infancy, and that even young children experience mourning when they suffer prolonged separation from or loss of their primary caregivers (Bowlby, 1960). As common as these ideas seem today, they were not widely accepted in his time. In attachment theory, the growing child's actual life experience, therefore, in particular, the child's early experiences with adequate care and, in unfortunate circumstances, the loss of a caregiver, are regarded as the most important determinants of development (Bowlby, 1982).

Bowlby also founded his theory on the evidence provided by comprehensive analyses and syntheses of scores of diverse ethological studies, with a particular concentration on primate studies, which he used to establish attachment theory within the larger theory of evolution. Attachment theory has been called a "major middle level" evolutionary theory (Simpson, 1999), because of its foundation in and refinement of Darwinian theory. Evolution theory became increasingly established as the dominant paradigm of the natural sciences in the $20^{\text {th }}$ century, though it had not, until Bowlby, had a significant place in our understanding of human psychosocial development. One of the great achievements, therefore, of Bowlby's attachment theory is that it represents the first time that a major theory of human psychosocial development became integrated into the dominant paradigm of modern biological sciences.

\section{LINKAGES OF ATTACHMENT THEORY TO HBSE CURRICULA IN SOCIAL WORK EDUCATION}

Attachment theory is a theory of human behavior within the social environment that incorporates the basic ideas of goal direction, exchange, and systemic integration characteristic of social systems theories. (See the summer 2004 issue of Development and Psychopathology for discussions of interfaces between attachment and social systems theories.) As Bowlby's basic notion of behavioral systems indicates (see discussion below), human behavior can only be understood as it becomes expressed in social relationship transactions. This view is thus representative of the contemporary view in developmental science that the old naturenurture dichotomy is a false one (National Research Council, 2000). What was formerly thought of as "nature" and "nurture" is now understood as component parts of one process; the one has no meaning without the other.

Attachment theory should be a cornerstone of theories presented in social work HBSE courses, because of its roots in human biology, its theoretical grounding in evolution theory, its explanatory power in addressing fundamental aspects of human experience, its reflection of social system dynamics, its widespread current acceptance among developmental scientists, and the vast empirical literature that supports it. While attachment theory has received notice in some HBSE texts, its presentation in them, with the notable exception of Haight and Taylor's recent text (2007), is often superficial. There are several current HBSE texts, however, that make no mention of attachment theory or of Bowlby. Surprisingly, many of the current HBSE texts still prominently feature detailed presentations of Freudian theory and its derivatives, despite the absence of empirical support for 
these and the view shared among the great majority of developmental scientists that, while of historical importance, as contemporary developmental theories they are obsolete. That the profession of social work, as judged by our HBSE texts, has taken so long to join the majority view of developmental scientists in this regard is puzzling.

There is a particular imperative for social workers and social work educators to be thoroughly grounded in attachment theory and research. As a profession, we have a unique responsibility to provide services to the most vulnerable of our society's children in our child welfare systems. Making the best-informed decisions about the well-being of these children requires thorough knowledge of the processes through which children form attachments to their caregivers. Unless we address these issues in depth in our curricula, reflecting the current state of knowledge in the field, we are neither honoring our professional responsibilities nor practicing ethically.

\section{COMPONENT PARTS OF ATTACHMENT THEORY}

\section{Theory of Instinctive Behavior: The Goal-Corrected Behavioral System Paradigm}

Attachment theory was first and foremost a new theory of human instinct and, thus, basic motivation. According to Bowlby, instinctive behavior necessarily involves the following four components: It is shared by a species; it contributes to individual or species survival; it is not dependent on learning; and it is characterized by a behavioral sequence, "not a simple response to a single stimulus" (1982, pg. 38). Despite the fact that his work to a great degree was concerned with the essential human instincts, he was wary toward the use of this term, concerned that it might communicate a view of inherited behavior operating in isolation from environmental conditions. Instead, borrowing a concept from cybernetics and control systems, Bowlby created the construct of the "goal-corrected behavioral system" to reflect the essential components of instinctive behavior as inherited capacities that are always dependent on the nature of the social context for their expression. Thus, the activation of behavioral systems in developing children always involves instinctive expression and the social learning associated with this in a feedback loop. The child influences this process through a capacity for "goal correction", that is, modification of behavior directed toward the social goal vis-àvis the caregiver, based on feedback received regarding the effectiveness of pursuit of the goal.

\section{Attachment Behavior}

Bowlby's main interest was the formation, beginning in infancy, of the behaviors that collectively compose the attachment behavioral system. Similar to the way in which the neonate responds to tactile stimulation of the lips and hard palate with reflexive grasping and sucking (elements of the feeding behavioral system), Bowlby proposed that the infant responds to conditions of vulnerability, such as fear, pain, cold, hunger, fatigue, and illness with reflexive behaviors, including crying, clinging, smiling, visually following the caregiver, signaling, and later, movement toward the caregiver. He proposed that the explanation for the genet- 
ic inheritance of these capacities lies specifically in their function in maintaining physical proximity to a caregiver. Beginning around the age of six months, these early behaviors become increasingly organized and incorporated into the attachment behavioral system (Bowlby, 1982). Proximity-seeking directed to a specific caregiver in distressing circumstances, the fundamental component of attachment behavior, serves the proximate purpose of calming feelings of insecurity and the evolutionary function to heighten the infant's odds of survival and, thus, the ultimate reproductive success of the species (Bowlby, 1982). The caregiver's sensitive response to the infant's activated attachment behavior produces, under favorable conditions, calming and a growing sense of emotional security.

\section{Attachment Behavior and Attachment}

Although Bowlby adapted terminology from cybernetics and control systems for his theory of instinct, the functioning of the attachment system is conceptualized primarily as an affective process, the chief consequence of which is the formation of a special class of affective bonds (what we think of as the attachment proper), which is a particular expression of love for a specific attachment figure and which tends to be relatively enduring across the lifespan. Because the formation of attachments is a life-long developmental theme (Ainsworth [1989] pointed out, for example, that successful marriages are those where the partners are able to alternate their reliance on each other as attachment figures), attachment theory recast what it means to need another person in times of distress. Anxiety and longing, as responses to separation from loved ones, could now be seen as normal indicators of strong bonds, not immaturity or insufficient autonomy.

\section{Attachment, Fear, and Exploration}

The attachment behavioral system functions directly in relation to two other major behavioral systems within the child (Bowlby, 1982), the fear/wariness and exploratory behavioral systems, representing, respectively, the instinct to withdraw from frightening circumstances and the instinct to explore novel situations. Bowlby proposed that the attachment and fear/wariness behavioral systems operate in close, but not absolute, synchrony. Very frequently, but not always, Bowlby argued, attachment behavior is activated by fear-inducing conditions that simultaneously activate withdrawal from the feared object. Attachment behavior may also be activated, however, according to Bowlby, in conditions not directly associated with an instinct to withdraw, such as fatigue and illness.

The exploratory behavioral system, in contrast to the fear/wariness system, plays a role fully equal in importance to the attachment system in determining how attachment behavior ultimately becomes expressed and, thus, how the attachment bond is formed. A natural, instinctive desire to explore the social and physical environment is normally activated in conditions where the child is relatively stress-free. Through this process, the child learns about the world, taking in new information and developing perceptual, analytical, and motor skills that provide opportunities for the child's development of mastery and autonomy. Inevitably, however, the child experiences depletion in energy or becomes distressed by encounters with unfamiliar objects or events, or from the separation from an attachment figure which produce felt vulnerability, activating the attach- 
ment behavioral system. In this way, exploratory and attachment systems are regarded as polar dimensions of a larger process, whereby the activation of one normally involves the relative deactivation of the other. In order for the child to be able to explore the world with confidence, s/he must be able to trust in the availability of the "secure base" of the attachment relationship, as described by Ainsworth (Ainsworth \& Bowlby, 1991), where the child can return for care and protection when needed. The way in which the attachment figure accurately perceives and responds to the child's alternating needs for exploration and attachment will determine the nature of the attachment bond that the child will form with the attachment figure. As Ainsworth (1989) later demonstrated, the transactions associated with exploration and proximity-seeking provide the infant with opportunities to learn an array of communication and social skills that become a foundation for social relatedness in ever widening social spheres as the infant matures into childhood. Essential relational styles of communication of need, and the expectations of others' responses to expressions of need, are therefore first learned in infancy in the first attachment relationships.

\section{Cognition and Development}

Through repeated experiences with the attachment figure in times of distress, the child learns about the responsiveness and reliability of the caregiving environment. These early experiences become increasingly organized in memory in structures Bowlby $(1973,1982)$ referred to as "internal working models", a term first used by the philosopher and cognitive psychologist Kenneth Craik. Bowlby preferred this term over more static representations of cognitive structures, because it communicates a sense of patterned responses to the social environment and, at the same time, the capacity for ongoing revision, based on learning from new social experiences. Internal working models, thus, can account for continuity of individual development as well as individual change. Internal working models become increasingly established and resistant to major revisions as the child grows, however. (The conceptualization of stability and change in cognition is one of several areas where Bowlby [1973] acknowledged the influence of Piaget on his thinking.) Early and consistent inadequate caregiving is especially associated with rigidly defensive representational models that are relatively impervious to change (Bowlby, 1980). Positive caregiving experiences tend to promote relatively flexible internal working models that serve a protective function for children: According to Bowlby, the primary adaptive function of internal working models is to provide the developing child with the capacity to predict the likely responses of potentially protective figures in situations where the child experiences vulnerability. Expectations of responsive care from others become associated with emotional security, which can provide a buffer against future negative interpersonal experiences (Weinfield, Sroufe, Egeland, \& Carlson, 1999).

From the initial period at age six months until approximately 18 months, Bowlby viewed the child's development of internal working models as embryonic. At this latter age, the child, under favorable conditions, is able to apply the representation of attachment figure(s) in a new capacity. The child now has the more complex mental organization of the internal working model of attachment figure increasingly available for the purpose of executing increasingly complex behav- 
ioral plans, especially those that involve venturing farther away from the proximity of the caregiver, $u$ sing the internal representation of the caregiver to make predictions about his/her availability (Bowlby, 1980).

As most children approach the end of the third year, the internal representation of the caregiver begins to carry with it the security-providing functions which, for the infant, were previously associated only with the actual presence of the attachment figure. At this point, the child can increasingly "turn to" the internal working model of the attachment figure when experiencing vulnerability when s/he is away from the attachment figure. Bowlby thought that this period is one in which the child is highly susceptible to external influences upon the formation of internal working models (Bowlby, 1980), reflecting their, as yet, formative nature.

While approximate developmental age-markers are useful for tracking these changes, it is important to note that Bowlby disliked the use of "watershed"-type age markers. He saw the development of internal working models as a gradual life process that changes qualitatively over time and is susceptible to revision. At the same time, however, according to his theory, it is the formation of internal working models in infancy that account for the enduring quality of early attachment relationships throughout life (Bowlby, 1982).

\section{The Formation of Internal Working Models in Unfortunate Conditions and} Defensive Processes

In optimal conditions, attachment figures respond adequately and consistently to children's experiences of vulnerability, and corresponding internal working models of attachment figures as reliable and trustworthy are formed. When a child consistently encounters behavior on the part of an attachment figure that does not adequately address conditions of vulnerability, however, the child is likely to form conflicting internal working models of the same attachment figure, a conscious internal working model that preserves a sense of the caregiver as good and one that is defensively excluded from consciousness containing the information regarding inadequate responses (Bowlby, 1973). Bowlby asserted that this sort of defensive exclusion is necessary for the child to preserve mental stability. Attempts to consciously integrate the experience of threat and persistent insecurity associated with an attachment figure would be likely to overwhelm the child. In order to preserve some sense of safety and security, the child maintains an illusory mental model of an adequate caregiver.

\section{Effects of Prolonged Separations from Caregivers}

Unlike nearly all other psychoanalysts of his time, Bowlby was especially interested in loss and the reactions of grief and mourning that young children between the ages of six months and three years experience when separated from their caregivers, the time of life when, as noted previously, attachment is especially sensitive to environmental changes. Relying to a great extent on Robertson's observational studies, Bowlby and Robertson (Bowlby, 1980; Robertson \& Bowlby, 1952) proposed that young children normally react to prolonged separation from their attachment figures in phases characterized by protest, despair, and (if the separation is not addressed) detachment. His theory of grief and mourning, influenced by the work of Colin Murray Parkes (Bowlby, 1980), involves a normative 
progression through four major phases of adjustment: a) numbing and shock; b) yearning and searching, accompanied by anger and disbelief; c) disorganization and despair, including the dismantling of internal working models of the relationships; and d) reorganization and redefinition. This theory proved to be very influential on the work of others in the field, including that of Kubler-Ross. In his theories of separation and loss, the phases of adjustment are characterized by initial activation and subsequent deactivation of attachment behavior for the missing or lost figure, thus the meaning and function of these events were recast as expressions of attachment behavior. He also emphasized that the course taken in childhood in reaction to separation or loss may well affect a person throughout the entire lifespan, depending to a great extent on the way in which attachment behavior was expressed in the relationship and the nature of response received from the caregiving environment.

\section{The Contribution to Attachment Theory of Mary Ainsworth and Colleagues}

In contrast to the comprehensive analytical syntheses and elegance of his theory, Bowlby directly participated to a lesser degree in empirical research. The application of his ideas about human development to empirical research was left by and large to his followers, the first and most notable of whom was Mary Ainsworth.

First in Uganda, and later in her landmark Baltimore study (Ainsworth, Blehar, Waters, \& Wall, 1978), home observations of mother and child interactions were coded for various aspects of the mother's sensitivity to the child and the child's autonomous and responsive behavior in relation to the mother. Patterns of secure and insecure attachment (described below) emerged from these observations, providing an empirical basis for Bowlby's theoretical construction of attachment in humans and expanding the theory of how individual differences occur with respect to the organization of attachment.

A major methodological innovation created by Ainsworth and colleagues in the Baltimore study was the so-called Strange Situation Procedure, a laboratory protocol containing several progressive steps designed to directly observe how infants react to separation from their attachment figures in an unfamiliar setting (see Solomon \& George, 1999, for more detail). Significant relationships were found between the home observations of the quality of mother-child interactions and the laboratory attachment patterns observed in the Strange Situation, suggesting that the laboratory observations were valid indicators of interactive behavioral patterns between mother and child.

The observations of mothers and children led to the construction of three main categories (composed of eight subgroups) reflecting what Ainsworth referred to as patterns of attachment. These categories are: Insecure-Avoidant (A); secure (B); and insecure-ambivalent (resistant) (C). In the Strange Situation, avoidant babies tended to avoid their mothers upon her return following separation by turning their backs or snubbing maternal overtures, and their communication styles were marked by affective restriction. Secure babies generally approached their mothers following separation and returned to exploration after having brief contact with her. Their communication styles were characterized by affective openness and mutuality, with clear modulation and regulation of extremes. 
Ambivalent babies tended to approach their mothers upon the mother's return following separations but remained dissatisfied and angry, not soothed by her presence. Their affective communication was typically disregulated and hostile.

Ainsworth's findings supported and elucidated several important aspects of Bowlby's theory of attachment, especially the notion that attachment behavior will look different for children depending on their life experiences. She made it clear, however, that these variations in attachment styles were not equivalent to variations in the strength of attachments (Ainsworth, 1972). Avoidant children, for example, are just as strongly attached to their caregivers as secure children; they differ in the strategies they have learned to express their attachment needs in relation to their specific caregivers.

The Strange Situation was important, first, because it provided a standard behavioral measure of attachment for infants up to approximately two years of age (it is typically used for infants between the ages of 12 and 18 months). It also came to be viewed as essentially a "shortcut" in the assessment of parent and child interaction that normally would be done in the naturalistic setting of the home (Ainsworth \& Marvin, 1995). Assessment in the Strange Situation, then, has become a standard against which other measures of attachment, especially those taking place later in childhood, are compared.

The work of Mary Main (Main \& Solomon, 1990), one of Ainsworth's many distinguished students, has contributed an additional fourth category of attachment behavior to Ainsworth's original formulation, the disorganized/disoriented category. Characteristics observed in the Strange Situation reunion behavior of children assigned to this group are immobilization and disorientation upon the mother's return, contradiction in physical movement, and, inferred from the contradictory physical movements, contradictions in intended or planned behavior. The disorganized/disoriented attachment pattern is considered a more severe adaptation to inadequate caregiving circumstances, distinguished by the absence of any coherent strategy for engagement and interaction, and has been shown to be prevalent among maltreated children (as high as $82 \%$ reported in Carlson, Cicchetti, Barnett, \& Braunwald, 1989).

\section{THE GOALS AND BOUNDARIES OF ATTACHMENT THEORY}

Bowlby clearly intended the attachment behavioral system to be understood as instinct-based behavior of at least equal importance to feeding and reproduction for lifespan development. This is not to say, however, that he viewed attachment in the same deterministic sense that Freudian theory reduced development to internal psychosexual drives. Instead, Bowlby conceptualized the formation of attachment in terms of dynamic systems that provide a foundation for the trajectory of lifespan development, which is modified through maturation and many other developmental influences, including subsequent relational interactions in the social ecology. 


\section{Other Important Influences on the Child-Parent Relationship and the Child's Personality}

\section{Attachment and Temperament}

In the early years of attachment research, there was some speculation that the individual differences that Ainsworth reported in attachment styles were actually temperamental differences (e.g., Kagan, 1982) and that, therefore, the theoretical edifice created by Bowlby was unnecessary to explain child development. Since then, however, several studies of attachment and temperament have concluded that these are independent processes (Belsky, 2005; Vaughn \& Bost, 1999) and that, while the child's temperament appears to exert some influence on the nature of the interaction that evolves between the child and his/her caregiver, the relational basis of the organization of individual differences in attachment has been shown to be robust over time and across cultures (van Ijzendoorn \& Sagi, 1999).

\section{Attachment and Social Learning}

Another area of early criticism of Bowlby's ideas came from adherents of social learning theories. From this viewpoint, of course, personality is regarded as the sum total of learning experiences, with essentially no allowance for any innate predispositions in the developing child beyond the seeking of pleasure and the avoidance of pain. Attachments form to caregivers, according to this viewpoint, as a secondary result of the gratification associated with meeting physiological needs. Attachment theory does not contradict the learning mechanisms associated with social learning theories, but it goes beyond them in its core emphasis on instinctive inheritance. Bowlby pointed out, for example, that children (as well as adults) form attachments to people who have nothing to do with providing sustenance or meeting other physiological needs, and he cited Harlow's primate studies, among many others, in support of his view (Bowlby, 1982). Likewise, as Ainsworth and Bowlby wrote, in circumstances where an infant is distressed, "timely and appropriate close bodily contact does not 'spoil' babies, making them fussy and clingy" (1991, pg. 7), a cautionary concern often voiced by traditional behaviorists.

\section{Attachment and Culture}

Like much of developmental research, many of the early studies of attachment, including Ainsworth's early Baltimore study, were done on white, middle-class samples in the U.S., with intact marriages. (The notable exception to this, as indicated above, was Ainsworth's very first fieldwork and the first empirical examination of attachment, which was done in Ugandan villages.) Since these early days, however, attachment has been studied in numerous cultures on every continent and across a wide diversity of environmental risk conditions. In general, the basic three attachment categories have proven to be robust indicators of children's development (van Ijzendoorn \& Sagi, 1999). Culture, however, does appear to exert an influence on the ways in which children come to express attachment and exploration in relation to their caregivers and, therefore, may influence the assessment of attachment in the Strange Situation. For example, in North American normative samples, approximately two-thirds of children are classified as secure and approximately one-fourth are classified as avoidant (the remainder 
are either insecure-ambivalent or disorganized). In contrast, research in Israel has found similar or higher percentages of secure children, more insecure-ambivalent children, and extremely few insecure-avoidant children. Despite variations such as these, the accumulated research has shown that attachment is a universal, normative phenomenon and that the major distinguishing factors among children's attachment classifications, as obtained in the SSP and other attachment assessments, relate to the quality of care and responsiveness provided by the caregiver and the social context within which this is provided (van Ijzendoorn \& Sagi, 1999).

\section{An Attachment Framework for Intervention}

Bowlby's original intention in constructing attachment theory was primarily to elucidate a practice theory that "fit the facts" better than Freudian theory. His ideas, however, were soon adopted by developmental research psychologists, who found in them powerful explanations for early childhood development. In recent years, there has been a renewed interest among many to return to Bowlby's original intention to apply the theory to problems associated with clinical intervention. In his later work, Bowlby (1988) described a set of guidelines for therapeutic intervention informed by attachment theory. Among these, he included the therapist's sensitivity to the primacy of safety and protection in the developmental experience of the client. Interpretations and analysis of the client's relational experience, including within the therapeutic relationship, should be based on an understanding of the centrality of the expression of attachment behavior to social experience and the creation of personal identity. Several evidenced-based interventions founded on attachment theory that incorporate these guidelines have recently been developed. These approaches focus first and foremost on systematic observations of parent-child interactions, many of which use videotape to document relationship qualities as well as teach parents how to strengthen their observational skills toward their children (Cohen et al., 2002; Lieberman, Silverman, \& Pawl, 2000; Marvin, Cooper, Hoffman, \& Powell, 2002; McDonough, 2004).

It is also important to note, however, that the construct of attachment and related concepts, unfortunately, have been distorted and misapplied to illegitimate interventions, often referred to as "attachment therapies." Part of this confusion of terms involves the notorious "holding therapy", variations of which have been responsible for serious injury and death (Boris, 2003; Mercer, 2001). Illegitimate uses of the concepts of attachment theory, which are perhaps inevitable, can only be avoided through true understanding of its tenets and related research findings.

\section{Emerging Ideas: Attachment and Developmental Psychopathology}

Recent advances in the field of developmental psychopathology have contributed to a new understanding of psychopathology more as adaptation than illness, and attachment theory has made a substantial contribution to this new direction of inquiry (Sroufe, 2005). Highlights of these new applications include recent work on Reactive Attachment Disorder (Lieberman \& Zeanah, 1995), new insights into the processes associated with traumatization and post-traumatic stress (LyonsRuth \& Jacobvitz, 1999; Main \& Hesse, 1990), and early attempts to discover developmental foundations for Axis II disorders based on attachment theory (Crittenden, 1997; Page, 2001a; Sroufe, 2005). 


\section{SELECTED MAJOR EMPIRICAL STUDIES}

Attachment theory created a virtual explosion of empirical research in developmental science over the past 40 years that continues to bring new discoveries in ever-increasing areas of inquiry. Due to space limitations, only a selection of this literature is reviewed here. For more detailed analyses, the reader is referred to Cassidy and Shaver's (1999) compendium.

The first and most important methodological contribution to the study of attachment remains the Ainsworth and Wittig Strange Situation Procedure (SSP) (see above), which is considered the "gold standard" of attachment research. Two of Ainsworth's students, Jude Cassidy and Robert Marvin, adapted the original SSP for use with preschool children up to age 5 (Cassidy \& Marvin, 1992). Since Ainsworth's landmark work, researchers following her trail have studied attachment in varied conditions with the SSP and newer methodologies, including various high-risk samples (Cicchetti \& Barnett, 1991; Crittenden, 1988; Maslin-Cole \& Spieker, 1990); culturally diverse samples (noted above); and in increasingly older samples, beyond infancy, the preschool and school years, and into adulthood (Bretherton, Prentiss, \& Ridgeway, 1990; Cassidy, 1988; Elicker, Englund, \& Sroufe, 1992; Feeney, 1999; Main, Kaplan, \& Cassidy, 1985; Sroufe, 1983; Sroufe, 2005). Children's attachment organization has been found to predict individual characteristics, such as self-esteem, emotional regulation, and cognitive and social competence (Weinfield, Sroufe, Egeland, \& Carlson, 1999; van Ijzendoorn \& Sagi, 1999), and it has been found to be predicted by qualities of the wider social ecology and history of the family, including mothers' sensitive responsiveness (Ainsworth, Blehar, Waters, \& Wall, 1978); mothers' social support (Crockenberg, 1981); parent-child communication qualities (Stewart \& Marvin, 1984; Sroufe, 1983; Main, Kaplan, \& Cassidy, 1985); and violence, maltreatment, and trauma (Lyons-Ruth \& Jacobvitz, 1999).

\section{Representational Research}

Most of the early studies of attachment relied on observed behavioral interactions of the child-caregiver dyads to determine how differences in security of attachment at one point in time relate to past dyadic interactions, concurrent social characteristics, or to future development or behavior. An important shift in the study of attachment occurred, however, when Main, Kaplan, and Cassidy (1985) suggested that the study of attachment should also be conducted at the representational level, directly assessing qualities of internal working models, especially as these are revealed in discourse qualities. In a pivotal longitudinal study, Main et al. (1985) examined the discourse qualities between 6 year-old children and their parents following a separation, as well as the parents' state of mind with respect to attachment as assessed with the Adult Attachment Interview (AAI; George, Kaplan, \& Main, 1984). The AAI is a semi-structured interview that probes for adults' memories, thoughts, and perceptions regarding their relationships with their own parents or attachment figures and how those relationships have affected their adult personalities and relationships with their own children. The interviews are scored on the basis of coherency of the discourse, involving qualities such as consistency, flow, truthfulness, and richness of supporting memories offered in responses, as well as the succinctness, completion, relevancy, and clar- 
ity of responses (Hesse, 1999). Of particular importance, these qualities of discourse, in contrast to the interview content, are the dimensions of most importance to the assessment of attachment organization with the AAI, because they reveal the ways in which significant memories of early experience are organized and stored (for more detail of the theory and method of the AAI, see Hesse, 1999). The interviews are classified into four categories, reflecting attachment styles, corresponding to the same attachment styles identified in children: autonomous/secure, dismissing/avoidant, preoccupied/ambivalent, and unresolved/disorganized. In the Main et al. study, secure parents were more likely to value attachment relationships, believe that attachment related experiences were influential on their own personality, and tell coherent, objective narratives, whereas dismissing parents minimized the value and importance of attachment relationships, preoccupied parents seemed confused or preoccupied with particular relationships, and unresolved/disorganized parents demonstrated lapses in discourse when discussing traumatic events or losses, indicating that they had not yet successfully resolved their mourning. Analyses indicated that these classifications of the parents' states of mind with respect to attachment were associated with their children's corresponding attachment classifications obtained in infancy (Main et al., 1985). Most impressively, adult attachment styles obtained with the AAI with expectant mothers in the third trimester of pregnancy have been found in subsequent studies to predict their children's attachment styles longitudinally when the children are 12 months old (Fonagy, Steele, \& Steele, 1991). The AAI has led to a new understanding of the significance of discourse qualities in speech and has spawned a new generation of interview protocols that reflect these insights (Oppenheim \& Koren-Karie, 2002; Zeanah \& Benoit, 1995).

Recent studies of attachment with foster parents and children using the AAI are of particular importance to social work. Among the salient findings of these studies, adoptive or foster parents' states of mind with respect to attachment are associated with the degree to which their foster homes provide developmentally corrective environments for maltreated children (Dozier, Higley, Albus, \& Nutter, 2002; Dozier, Stoval, Albus \& Bates, 2001).

\section{Children's Representations}

Several representational measures of young children's attachment organization have also been developed in recent years using semi-projective techniques. For example, George, Kaplan, \& Main (1985) used the Separation Anxiety Test (SAT) to elicit children's narratives about drawings illustrating child-parent separations and derived coded attachment categories that were found to be associated with their infant attachment classifications obtained from Strange Situations.

Narrative Story Stem Techniques (NSST; Bretherton, Ridgeway, \& Cassidy, 1990; Emde, Wolf, \& Oppenheim, 2003) are similar to the SAT in their projective natures but use play props to facilitate the child's narrations and increase the interpretability of the child's representations. In 20 years of research, the NSST has been found to relate to several dimensions of children's and parents' psychological and social functioning, including infant attachment, child social competence, child maltreatment, and parent psychological distress (see Page, 2001b for a review). Recently (Toth, Maughan, Todd-Manly, Spagnola, \& Cicchetti, 2002), the 
NSST has also been used as an outcome variable for a parenting intervention, showing that clinical improvement on the part of parents is associated with children's attachment representations of children in relation to parents in predicted ways.

\section{Attachment and Development Over the Lifespan}

Attachment research has also been conducted in the lives of participants in several longitudinal studies over the course of several years (see Grossmann, Grossmann, \&Waters, 2005). The first of these, the Minnesota Longitudinal Study of Parents and Children, merits special consideration, however brief, because of its unique contribution to our understanding of the linkages between infantcaregiver relationships and child development. This study began in 1975 with the recruitment of 267 low-income, first-time mothers in their third trimester of pregnancy and continues today with the children now in their 30s. Numerous assessments have been made, including representational and observational, across home, laboratory, and school settings, to examine the predictive power of attachment across the lifespan. In brief, the quality of attachment relationships established in infancy, especially when combined with other conditions in the family social ecology, have been shown to be predictive of children's developmental outcomes, particularly in the areas of self-reliance, emotional self-regulation, cognitive and social competence, and in particular for disorganized attachments, psychopathology (Sroufe, 2005). This and other longitudinal studies provide support for Bowlby's notion that the "trajectories" of development, once established, are relatively difficult to alter (Vaughn, 2005, p. 378).

\section{NEXT STEPS FOR THEORY PROGRESSION AND IMPLICATIONS FOR SOCIAL WORK}

In 1999, Mary Main contributed an essay for the Handbook of Attachment entitled, Attachment Theory: Eighteen Points with Suggestions for Future Studies, in which she provided a comprehensive discussion of current issues in theory and research development. Using this as a foundation, we identify here a selection of four issues that we consider important for future exploration and application of theory.

\section{Integration of Multiple Attachments/Attachment Hierarchies}

Bretherton (1985), in expanding on Bowlby's original ideas, proposed that internal working models eventually become organized in hierarchical structures, where, in some instances, the model derived from one attachment relationship supersedes others in providing guidance and direction and, in other instances, syntheses of internal working model qualities are formed. Bowlby used the term "monotropy" to describe the tendency for a developing child to regard one attachment figure above all others as primary. There has been little direct empirical investigation of these ideas, and much more needs to be learned, especially in circumstances where children live with multiple attachment figures.

\section{The Critical Period for Attachment Formation}

Bowlby's original theory posited that the first three years mark the critical period for the initial formation of attachment in normal circumstances and subsequent research has supported this (Main, 1999). Despite some recent groundbreaking studies of previously institutionalized children (see Zeanah, Smyke, Koga, \& 
Carlson, 2005), little is yet known about how children cope with the most extreme adversity in caregiving environments and their capacity for the formation of attachments relatively later in childhood. Consequently, more needs to be known about how children form so-called "second" attachments, as in the case of placement in foster homes. A child's capacity to form an attachment after suffering severe deprivation is likely to be the single most important developmental event in the child's life, setting the stage for future social relatedness.

\section{Brain Chemistry}

Main pointed out that a more precise understanding of the function of fear in relation to attachment must include a better understanding of the neurochemical processes and brain functions, in particular the role of the amygdala involved in the activation of the attachment behavioral system. Bowlby posited that attachment behavior is founded in a genetically inherited capacity controlled by the central nervous system. An as yet unknown portion of variation in the way in which attachment becomes expressed may be associated with differences in brain chemistry and functions.

\section{Assessment and Intervention}

Perhaps most important to social work, far more work must be done to integrate attachment theory and research methods into social work practice. We believe that there are, in particular, four important areas where this can be accomplished: Assessments with children, especially adapting representational measures such as the NSST to clinical settings; adult interviewing, using the insights of research with the AAI on the significance of discourse qualities to attachment organization; the use of videotape, using the technical achievements from the history of observational attachment research; and education for parents and foster parents, applying our developmental knowledge about the significance of attachment to development to problems in parenting. In particular, many commonly observed problems encountered in clinical interventions concerning family violence and child maltreatment, such as the strong, instinctive desire to seek and maintain proximity to an abuser, are illuminated with an understanding of attachment behavioral dynamics and the imperative for survival associated with violent circumstances (Bowlby, 1982; Page, 1999). In general, much more needs to be learned, and what is currently known disseminated more broadly, about the centrality of the human need for safety and security as central organizing concepts in clinical assessment, diagnosis, treatment planning, and intervention. The understanding of basic needs as embodied in attachment theory, in our view, should be core to the conceptualization of treatment approaches.

The past 40 years have brought us considerable new knowledge about the importance for lifespan development of the processes associated with attachment organization. We in the profession of social work are now challenged to take this knowledge and effectively incorporate it into our educational and practice methods to better respond to the needs of vulnerable children and families. 


\section{References}

Ainsworth, M.D.S. (1972). Attachment and dependency: A comparison. In J.L. Gewirtz (Ed.), Attachment and dependency (pp. 97-137). Washington, D.C.: V.H. Winston.

Ainsworth, M.D.S. (1989). Attachments beyond infancy. American Psychologist, 44, 709-716.

Ainsworth, M.D.S., Blehar, M.C., Waters, E., \&Wall, S. (1978). Patterns of attachment: A psychological study of the strange situation. Hillsdale, N.J.: Erlbaum Associates.

Ainsworth, M.D.S., \& Bowlby, J. (1991). An ethological approach to personality development. American Psychologist, 46(4), 333-341.

Ainsworth, M.D.S., \& Marvin, R.S. (1995). On the shaping of attachment theory and research: An interview with Mary D.S. Ainsworth. Monographs of the Society for Research in Child Development, 60(2-3), pp. 321.

Belsky, J. (2005). Attachment theory and research in ecological perspective: Insights from the Pennsylvania Infant and Family Development Project and the NICHD Study of Early Child Care. In K. Grossmann, K. Grossmann, \& E. Waters (Eds.), Attachment from infancy to adulthood: The major longitudinal studies (pp. 71-97). New York: Guilford Press.

Boris, N.W. (2003). Attachment, aggression and holding: A cautionary tale. Attachment \& Human Development, 5(3), 245-247.

Bowlby, J. (1960). Grief and mourning in infancy and early childhood. The Psychoanalytic Study of the Child. 15, 9-52.

Bowlby, J. (1973). Attachment and loss, Vol. II: Separation: Anxiety and anger. New York: Basic Books.

Bowlby, J. (1980). Attachment and loss, Vol. III: Loss: Sadness and depression. New York: Basic Books.

Bowlby, J. (1982). Attachment and loss, Vol. I: Attachment (2 ${ }^{\text {nd }}$ ed.). New York: Basic Books.

Bowlby, J. (1988). Attachment, communication, and the therapeutic process. In J. Bowlby, A secure base (pp. 137-157). New York: Basic Books.

Bretherton, I. (1985). Attachment theory: Retrospect and prospect. In I. Bretherton \& E. Waters (Eds.), Growing points of attachment theory and research. Monographs of the Society for Research in Child Development, 50, Serial no. 209 (1-2), 3-35.

Bretherton, I., Ridgeway, D., \& Cassidy, J. (1990). Assessing internal working models of the attachment relationship: An attachment story completion task for 3-year-olds. In M.T. Greenburg, D. Cicchetti, \& E.M. Cummings (Eds.), Attachment in the preschool years (pp. 273-308). Chicago, IL: University of Chicago Press.

Bretherton, I., Prentiss, C., \& Ridgeway, D. (1990). Children's representations of family relationships in a story completion task at 37 and 54 months. In I. Bretherton \& M. Watson (Eds.), Children's perspectives on the family: New directions in child development (series), vol. 48 (pp. 85-105). San Francisco: JosseyBass.

Carlson, V., Cicchetti, D., Barnett, D., \& Braunwald, K. (1989). Disorganized/disoriented attachment relationships in maltreated infants. Developmental Psychology, 25, 525-531.

Cassidy, J. (1988). Child-mother attachment and the self in six year-olds. Child Development, 59(1), 121-134.

Cassidy, J., \& Marvin, R.S. (1992). Attachment organization in preschool children: Procedures and coding manual ( $5^{\text {th }}$ ed.). Unpublished manuscript, MacArthur Working Group on Attachment, Seattle, WA.

Cassidy, J., \& Shaver, P.R. (Eds.) (1999). Handbook of attachment: Theory, research, and clinical applications. New York: Guilford Press.

Cicchetti, D., \& Barnett, D. (1991). Toward the development of a scientific nosology of child maltreatment. In W. Grove \& D. Cicchetti (Eds.), Thinking clearly about psychology: Vol. 2, Personality and psychopathology (pp. 346-377). Minneapolis: University of Minnesota Press.

Cohen, N.J., Lojkasek, M., Muir, E., Muir, R., \& Parker, C.J. (2002). Six-month follow-up of two motherinfant psychotherapies: Convergence of therapeutic outcomes. Infant Mental Health Journal, 23(4), 361-380. 
Crittenden, P.M. (1997). Truth, error, omission, distortion, and deception: The application of attachment theory to the assessment and treatment of psychological disorder. In S.M. Clancey Dollinger \& L.F. DiLalla (Eds.). Assessment and intervention: Issues across the life-span (pp. 35-76). Mahwah, NJ: Lawrence Erlbaum.

Crittenden, P. (1988). Relationships at risk. In J. Belsky \& T. Nezworski (Eds.), Clinical implications of attachment (pp. 136-174). Hillsdale, NJ: Erlbaum.

Crockenberg, S.B.M. (1981). Infant irritability, mother responsiveness, and social support influences on the security of infant-mother attachment. Child Development, 52(3), 857-866.

Dozier, M., Higley, E., Albus, K.E., \& Nutter, A. (2002). Intervening with foster infants' caregivers: Targeting three critical needs. Infant Mental Health Journal, 23(5), 541-554.

Dozier, M., Stoval, K.C., Albus, K.E., \& Bates, B. (2001). Attachment for infants in foster care: The role of caregiver state of mind. Child Development, 72(5), 1467-1477.

Elicker, J., Englund, M., \& Sroufe, L.A. (1992). Predicting peer competence and peer relationships in childhood from early parent-child relationships. In R. Parke and G. Ladd (Eds.), Family-peer relationships: Modes of linkage (pp. 77-106). Hillsdale, NJ: Erlbaum.

Emde, R.N., Wolf, D.P., \& Oppenheim, D. (Eds.)(2003). Revealing the inner worlds of young children: The MacArthur story stem battery and parent-child narratives. New York: Oxford University Press.

Feeney, J.A. (1999). Adult romantic attachment and couple relationships. In J. Cassidy \& P. Shaver (Eds.), Handbook of attachment: Theory, research, and clinical applications (pp. 355-377). New York: Guilford Press.

Fonagy, P., Steele, H., \& Steele, M. (1991). Maternal representation of attachment during pregnancy predict the organization of infant-mother attachment at one year of age. Child Development, 62, 891-905.

George, C., Kaplan, N., \& Main, M. (1984). Adult Attachment Interview protocol. Unpublished manuscript, University of California at Berkley.

Greenberg, J.R., \& Mitchell, S.A. (1983). Object relations in psychoanalytic theory. Cambridge, MA: Harvard University Press.

Grossmann, K., Grossmann, K., \& Waters, E. (Eds.) (2005). Attachment from infancy to adulthood: The major longitudinal studies. New York: Guilford Press.

Haight, W.L., \& Taylor, E.H. (2007). Human behavior for social work practice: A developmental-ecological framework. Chicago: Lyceum Books.

Hesse, E. (1999). The Adult Attachment Interview. In J. Cassidy \& P.R. Shaver (Eds.), Handbook of attachment: Theory, research, and clinical applications (pp. 395-433). New York: The Guilford Press.

Kagan, J. (1982). Psychological research on the human infant: An evaluative summary. New York: W.T. Grant Foundation.

Lieberman, A.F., Silverman, R., \& Pawl, J.H. (2000). Infant-parent psychotherapy: Core concepts and current approaches. In C.H. Zeanah (Ed.), Handbook of infant mental health ( $2^{\text {nd }}$ ed.) (pp. 472-484). New York: Guilford Press.

Lieberman, A., \& Zeanah, C.H. (1995). Disorders of attachment in infancy. In K. Minde (Ed.), Infant psychiatry, child psychiatric clinics of North America (pp. 571-588). Philadelphia: W.B. Saunders.

Lyons-Ruth, K., \& Jacobvitz, D. (1999). Attachment disorganization: Unresolved loss, relational violence, and lapses in behavioral and attentional strategies. In J. Cassidy \& P. Shaver (Eds.), Handbook of attachment: Theory, research, and clinical applications (pp. 520-554). New York: Guilford Press.

Main, M. (1999). Attachment theory: Eighteen points with suggestions for future studies. In J. Cassidy \& P. Shaver (Eds.), Handbook of attachment: Theory, research, and clinical applications (pp. 845-887). New York: Guilford Press.

Main, M., \& Hesse, E. (1990). Parents' unresolved traumatic experiences are related to infant disorganized attachment status: Is frightened and/or frightening parental behavior the linking mechanism? In M.T. Greenberg, D. Cicchetti, \& E.M. Cummings (Eds.), Attachment in the preschool years: Theory, research, and intervention (pp. 161-182). Chicago: University of Chicago Press.

Main, M., Kaplan, K., \& Cassidy, J. (1985). Security in infancy, childhood and adulthood: A move to the level of representation. In I. Bretherton \& E. Waters (Eds.), Growing points of attachment theory and research, monographs of the Society for Research in Child Development, 50, Serial No. 209 (1-2), 66-104. 
Main, M., \& Solomon, J. (1990). Procedures for identifying infants as disorganized/disoriented during the Ainsworth Strange Situation. In M.T. Greenberg, D. Cicchetti, \& E.M. Cummings (Eds.), Attachment in the preschool years: Theory, research, and intervention (pp. 121-160). Chicago: University of Chicago Press.

Marvin, R., Cooper, G., Hoffman, K., \& Powell, B. (2002). The Circle of Security Project: Attachment-based intervention with caregiver-preschool child dyads. Attachment and Human Development, 4(1), 107-124.

Maslin-Cole, C., \& Spieker, S.J. (1990). Attachment as a basis for independent motivation: A view from risk and nonrisk samples. In M.T. Greenberg, D. Cicchetti, \& E.M. Cummings (Eds.), Attachment in the preschool years: Theory, research, and intervention (pp. 245-272). Chicago: University of Chicago Press.

McDonough, S. (2004). Interaction guidance: Promoting and nurturing the caregiving relationship. In A. Sameroff, S. McDonough, \& K. Rosenblum (Eds.), Treating parent-infant relationship problems: Strategies for intervention (pp. 79-96). New York: Guilford Press.

Mercer, J. (2001). Attachment therapy using deliberate restraint: An object lesson on the identification of unvalidated treatments. Journal of Child and Adolescent Psychiatric Nursing, 14(3), 105-114.

National Research Council and Institute of Medicine (2000). From neurons to neighborhoods: The science of early childhood development. Committee on Integrating the Science of Early Childhood Development. J.P. Shonkoff \& D.A. Phillips (Eds.). Board of Children, Youth, and Families, Commission on Behavioral and Social Sciences and Education. Washington, D.C.: National Academy Press.

Oppenheim, D., \& Koren-Karie, N. (2002). Mothers' insightfulness regarding their children's internal worlds: The capacity underlying secure child-mother relationships. Infant Mental Health Journal, 23(6), 593-605.

Page, T. (1999). The attachment partnership as conceptual base for exploring the impact of child maltreatment. Child \& Adolescent Social Work Journal, 16(6), 419-437.

Page, T. (2001a) Attachment and personality disorders: Exploring maladaptive developmental pathways. Child \& Adolescent Social Work Journal, 18(5), 313-334.

Page, T. (2001b). The social meaning of children's narratives: A review of the attachment-based narrative story stem technique. Child and Adolescent Social Work Journal, 3, 171-187.

Robertson, J., \& Bowlby, J. (1952). Responses of young children to separation from their mothers. Courrier du Centre International de l'Enfance, 2, 131-142.

Robertson, J., \& Robertson, J. (1959). A two year-old goes to hospital [Film]. Tavistock Child Development Research Unit, London (available through Penn State Media Sales, Pennsylvania State University, University Park, PA).

Simpson, J.A. (1999). Attachment theory in modern evolutionary perspective. In J. Cassidy \& P. Shaver (Eds.), Handbook of attachment: Theory, research, and clinical applications (pp. 115-140). New York: Guilford Press.

Solomon J, \& George C (1999). The measurement of attachment security in infancy and childhood. In J. Cassidy \& P.R. Shaver (Eds.). Handbook of attachment: Theory, research, and clinical applications (pp. 287-316). New York: Guilford Press.

Sroufe, L.A. (1983). Infant-caregiver attachment and patterns of adaptation in preschool: The roots of maladaptation and competence. In M. Perlmutter (Ed.), Minnesota Symposium in Child Psychology, 16, 4191. Hillsdale, NJ: Erlbaum.

Sroufe, L.A. (2005). Attachment and development: A prospective, longitudinal study from birth to adulthood. Attachment \& Human Development, 7(4), 349-367.

Stewart, R.B., \& Marvin, R.S. (1984). Siblings relations: The role of conceptual perspective taking in the ontogeny of sibling caregiving. Child Development, 55(4), 1322-1333.

Toth, S.L., Maughan, A., Todd Manly, J., Spagnola, M., \& Cicchetti, D. (2002). The relative efficacy of two interventions in altering maltreated preschool children's representational models: Implications for attachment theory. Development and Psychopathology, 14, 877-908.

van Ijzendoorn, M.H., \& Sagi, A. (1999). Cross-cultural patterns of attachment: Universal and contextual dimensions. In J. Cassidy \& P. Shaver (Eds.), Handbook of attachment: Theory, research, and clinical applications (pp. 713-734). New York: Guilford Press. 
Vaughn, B.E. (2005). Discovering pattern in developing lives: Reflections on the Minnesota study of risk and adaptation from birth to adulthood. Attachment and Human Development, 7(4), 369-380.

Vaughn, B.E., \& Bost, K.K. (1999). Attachment and temperament. In J. Cassidy \& P. Shaver (Eds.), Handbook of attachment: Theory, research, and clinical applications (pp. 198-225). New York: Guilford Press.

Weinfield, N.S., Sroufe, L.A., Egeland, B., \& Carlson, E. (1999). The nature of individual differences in infant-caregiver attachment. In J. Cassidy \& P. Shaver (Eds.), Handbook of attachment: Theory, research, and clinical applications (pp. 68-88). New York: Guilford Press.

Zeanah, C.H., \& Benoit, D. (1995). Clinical applications of a parent perception interview. Child and Adolescent Psychiatric Clinics of North America, 4(3), 539-553.

Zeanah, C.H., Smyke, A.T., Koga, S.F., \& Carlson, E. (2005). Attachment in institutionalized and community children in Romania. Child Development, 76(5), 1015-1028.

\section{Author's Note:}

Address correspondence to: Timothy Page, M.S.W., Ph.D., Louisiana State University School of Social Work, 216 H.P. Long Fieldhouse, Baton Rouge, LA 70803, USA. e-mail: tpage2@Isu.edu. 